Letter to the Editor

\title{
Drug abuse among nurses: A neglected challenge
}

\author{
Fereshteh Araghian Mojarad ${ }^{1}$, Leila Jouybari², Akram Sanagoo ${ }^{3, *}$
}

(Received: 22 Mar 2016; Accepted: 18 Apr 2016)

Drug dependence is a prominent issue in individuals of different professions, education levels, and socioeconomic classes. Authors of this paper, including the first author (as a qualified clinical nurse and nursing supervisor), aimed to address the challenges associated with substance abuse and the subsequent failure in interactions between healthcare personnel and patients referring to clinical centers.

Clinical reports have denoted various forms of addiction as a common problem among clinicians. In this regard, a nursing shift supervisor remarks: In one of my shifts, I noticed that a fellow paramedic member had a long list of prescription drugs, which seemed unreasonable. After checking the prescription, I reported the matter to the matron, who mentioned that the majority of paramedic staff members suffered from substance abuse during their shifts."

The quotation above suggests that in addition to nurses, drug addiction affects different healthcare team members. Unfortunately, reported cases of drug dependence in the clinical environment are frequently neglected by hospital authorities, leading to the failure of rehabilitation attempts. Furthermore, substance abuse among healthcare personnel may cause disabilities, failure in clinical performance, expulsion, early retirement, and even death. The concept of "addiction potential" suggests that in case of availability, some individuals are more prone to substance abuse than others (1). This issue has prevailed among nurses for at least 150 years.

According to historical evidence, reports of drug addiction among nurses emerged in the mid-19th century during the era of Florence Nightingale. Moreover, the American Nurses Association reported substance-related disorders among nurses in 1980 for the first time (2). In a cross-sectional study performed in a medical school in India, 229 male physicians, 1,130 medical male students, and 73 female nursing students were reported to be drug abusers (3). Furthermore, approximately $10-15 \%$ of all healthcare professionals experience substance abuse at a certain point in their professional life. Identification of healthcare professionals with drug abuse is more complicated compared to the general population (2). Findings of a study indicated that the prevalence of substance abuse among nurses and general population is about $10 \%$ (4).

Drug dependence is a grave concern, likely to disintegrate the standards of nursing practice and delivery of safe, high-quality care (5). Therefore, nurses with substance abuse problems must receive special attention and support since they might risk the health of patients. However, such consequences may be undermined due to the refusal to report substance abuse issues in treatment centers. In healthcare team members with drug dependence, fear of punishment could be an obstacle for requesting assistance or cooperation in rehabilitation (2).

Based on the systematic observation and clinical

\footnotetext{
${ }^{1}$ Student Research Committee, Golestan University of Medical Sciences, Gorgan, Iran

${ }^{2}$ Nursing Research Center, Goletsan University of Medical Sciences, Gorgan, Iran

3,* Corresponding author: Nursing Research Center, Golestan University of Medical Sciences, Gorgan, Iran. Email: sanagoo@goums.ac.ir
} 
experience of the authors of this article, since drug addiction is a social stigma, almost all healthcare personnel refuse to report such cases to the related authorities. Other challenges in this regard are lack of appropriate instructions to prevent substance abuse in treatment centers and insufficiency of comprehensive studies on drug addiction among medical staff, especially nurses. Therefore, national research is required regarding the prevalence and influential factors in substance abuse among healthcare providers. In addition, basic strategies must be adopted as to tackle this issue in clinical environments.

With their remarkable professional reputation in the community, medical personnel are responsible for the safety and welfare of patients. As such, healthcare providers with drug abuse should be supported through non-punitive approaches, early intervention, and referral to specialists in order to receive effective treatment. To overcome this silent epidemic and prevent the devastation of organizational capital, a few strategies could be adopted, including a National Awareness Day (Drug addiction is an illness, not a crime), in-service education of healthcare staff, periodic training of nursing directors and hospital managers, identification of staff with drug abuse and providing support facilities, and proper management of drug storage and usage in hospitals.

\section{References}

1. Agha Yosefi A, Safarinia M, Abbaspour P. The investigating of drug addiction potential among medical students: role of subjective components of anger. J Res Addict 2015; 9(35):25-35 (Persian).

2. Hiremath P. Alcohol addiction among nurses. J Comm Pub Health Nurs 2015; 1(1):106.

3. Mohan S, Pradeepkumar AS, Thresia CU, Thankappan KR, Poston WS, et al. Tobacco use among medical professionals in Kerala, India: the need for enhanced tobacco cessation and control efforts. Addict Behav 2006; 31(12):2313-8.

4. Dunn D. Substance abuse among nurses-defining the issue. AORN J 2005; 82(4):573-82.

5. Thrinkoff AM, Storre CL. Substance use among nurses: differences between specialties. Am J Public Health 1998; 88(4):581-5. 